\title{
Candidate genes and biological processes in de novo CNVs from autistic individuals
}

\author{
Hyun J Noh ${ }^{1 *}$, Christian R Marshall ${ }^{2}$, Dalila Pinto ${ }^{2}$, Stephen W Scherer ${ }^{2}$, Chris P Ponting ${ }^{1}$, Caleb Webber ${ }^{1}$ \\ From Beyond the Genome: The true gene count, human evolution and disease genomics \\ Boston, MA, USA. 11-13 October 2010
}

\section{Background}

There is growing evidence that common diseases might not result from a few common variants, but rather from many rare variants across many genes/loci. This is likely to be particularly true for disorders such as autism where the fecundity of affected individuals is exceptionally low $(\sim 5 \%)$ and thus disease-predisposing alleles are unlikely to be transmitted. Since these different disease-contributing genes give rise to a shared disease phenotype, it is likely that their protein products contribute to shared biological processes, such as particular protein pathways or cellular networks. Identifying the common network(s) among these genes would allow the screening of a small subset of genes for early diagnosis and these networks could present multiple points for early intervention.

\section{Methods}

We examined two independent sets of de novo copy number variants (CNVs) identified in individuals with autism spectrum disorders (ASDs): (i) a large discovery set of 73 CNVs identified in 54 patients (median size $118 \mathrm{~Kb}$ ) from the Autism Genome Project (AGP) consortium [1]; and (ii) a small validation/replication set of $28 \mathrm{CNVs}$ identified in 24 patients (median size $3 \mathrm{Mb}$ ) [2]. To identify unexpected shared features among genes overlapped by these CNVs, we exploited phenotype data arising from the disruption of $\sim 6000$ human orthologues in mouse. We infer that genes are likely to act in the same biological process if, when disrupted in mouse, they exhibit comparable phenotypes. Thus, we aim to identify significantly over-represented mouse phenotypes among sets of human genes of interest (e.g. genes overlapped by autism-associated CNVs) [3].

'MRC Functional Genomics Unit, University of Oxford, Oxford, OX1 3QX, UK Full list of author information is available at the end of the article

\section{Results}

Among genes overlapped by the AGP CNVs, significant enrichments of specific mouse phenotypes were discovered $(\mathrm{FDR}<5 \%)$, most of which were either replicated $(\mathrm{FDR}<5 \%)$ or validated $(p<0.05)$ in the Marshall set, including: (i) A significant enrichment of genes associated with four abnormal synaptic transmission phenotypes; (ii) A significant enrichment of genes associated with $>10$ behavioural phenotypes and five hearing phenotypes that are only found within duplicated CNVs. Many of these duplication-specific phenotypic associations are readily comparable to ASD symptoms, such as abnormal motor learning $(60-80 \%$ of individuals with ASD exhibit impaired coordination), nonconvulsive seizures ( $1 / 3$ of individuals with ASD suffer seizures, commonly non-convulsive) and sensorineural hearing impairment (25-40\% individuals with ASD have hearing impairments). These enrichments identify $>100$ largely novel candidate genes and provide causal hypothesis for between $19-83 \%$ of patients in each cohort.

\section{Author details}

${ }^{1}$ MRC Functional Genomics Unit, University of Oxford, Oxford, OX1 3QX, UK. ${ }^{2}$ The Centre for Applied Genomics and Program in Genetics and Genomic Biology, The Hospital for Sick Children and Department of Molecular Genetics, University of Toronto, Ontario, M5G 1L7, Canada.

Published: 11 October 2010

\section{References}

1. Autism Genome Project Consortium: Functional impact of global rare copy number variation in autism spectrum disorder. Nature 2010, doi:10.1038/ nature09146.

2. Marshall, et al: Structural variation of chromosomes in autism spectrum disorder. AJHG 2008, 82:477-88.

3. Webber, et al: Forging links between human mental retardationassociated CNVs and mouse gene knockout models. PLOS Genet 2009, 5(6):e1000531.

doi:10.1186/1465-6906-11-S1-O9

Cite this article as: Noh et al:: Candidate genes and biological processes in de novo CNVs from autistic individuals. Genome Biology 2010 11(Suppl 1):09. 